\title{
COMPUTATIONAL DESIGN USED FOR JEWELRY
}

\author{
Nikolaos Efkolidis (D), Prodromos Minaoglou (D), Kyriaki Aidinli (D), Panagiotis Kyratsis $(\mathbb{1}$ \\ University of Western Macedonia, Department of Product and Systems Design Engineering, \\ Kozani, Greece
}

\begin{abstract}
Nowadays more and more computer-aided technologies and computational techniques are applied to product design. Jewelry belongs to those products. Jewelry is considered as part of human civilization and adopted to human's needs. In recent years, computational design is applied in order to overcome the limitations of traditional CAD systems, when reaching an increased level of complexity and geometrical controls. Computational design supports the evolution of creative design and develops models as a form of the computational exploration. Furthermore, additive manufacturing (AM) pieces of equipment are widely used to create models and prototype parts in various applications. AM plays an important role, when developing jewelry models, as it is utilized with an aim to produce high-quality models in rather reduced numbers and complex shapes. In the current paper, a computer-based design tool was applied in order to develop algorithms for the generation of new artistic forms for the design of a set of jewelry. Then, Stereolithography (SLA) technique is chosen for the prototype models construction.
\end{abstract}

Key words: Computational design, Jewelry design, Computer-aided-design, Additive Manufacturing

\section{INTRODUCTION}

Jewelry is one of the oldest types of products, according to the findings which are dating from almost 100,000 years ago. It is considered to be a human need and part of human civilization. Its creation depends on cultural and social impacts of a particular period. There are many types of jewelry, which is used for the decoration on human's body, such as, rings, necklaces, bracelets, earrings, brooches, etc. (Silina et al, 2015; larussi et al, 2015; Manavis et al, 2020a). So, a large variety of forms were designed and developed driven by the available materials and technology at the time. Jewelry is an industrial design product which combine aesthetics, ergonomics and usability. So, the design process involves investigation and creativity, and its development in the case of mass production is a combination of art, science, and engineering. Nowadays, the most commercially available jewelry production uses Computer-Aided Design (CAD) and manufacturing technology. Several Computer-Aided Design pieces of software have been developed to help the designers with an aim to produce quality products in shorter time-to-market.

Somlak et al (2011) proposed an aesthetic-driven evolutionary algorithm based on a genetic algorithm (GA) for fractal-based user-centered jewelry design. A two-step fitness function was created in order to determine morphology and aesthetics of the generated art forms. Human preferences Surveys, and popular long-lasting symbols were used for the creation of the aesthetic evolutionary algorithm. They reached a conclusion that the proposed GA can increase design productivity by about $80 \%$. Goel et al (2015) used Computer Aided Geometric Modeling for the recreation of traditional ornamental designs to innovate the designs due to new interpretations of the traditional styles. The basic concept was the development of mathematical models and algorithms for the design of traditional Indian jewelry by simplifying the design methodology of complex traditional ornamental designs. Miguel et al (2016) presented a computational method for designing wire sculptures consisting of interlocking wires. The computation of aesthetically pleasing wire sculptures that are structurally stable, present an extremely efficient and fast alternative for low-fidelity rapid prototyping. A similar research was done by Wang et al (2019) as they introduced a computer assisted framework for manually creating 3D wire bending art from given 3D models. They developed a method, which extracts a set of 3D contour-curves from several viewpoints as a target design of wire sculpture. Tian et al (2019) explored an efficient customization method to adapt the user's need for flexible and custom design of shoes. All the characteristic parameter and related algorithm combined with the formation characteristics of the shoe last bottom map and analysed. The parametric design was used to construct the automatic moulding program of the shoe last bottom to form a professional and feasible design method. The result was the improvement of the design and production efficiency of the customized shoe last bottom. Shen et al (2018) used computational design in order to adjust the three-dimensional structure of clothing and garment. They established garment pieces as the basic modeling form, which conforms to the perfect curvature of the human body. The result was to manage change of the logical structure for the new form of garment construction. 
In the current paper, it is presented a computer-aided fabrication approach, that was designed to make jewelry production easier and faster and to increase the popularity of hand-crafting objects by making them readily available. Our main contribution is the integration of jewelry techniques with a digital design system to produce extremely unusual forms compared with preceding methods. Computational design at the same time with jewelry design and 3D printing, suggests a different approach. The workflow used in this work is simply schematized in Figure. 1. The design steps include the conceptual stage with CAD based sketches developed and then forward the outcome to advanced computational tools for producing a series of alternative designs (family of products by altering the geometrical parameters introduced) and finally the proposed one. This is used at the next stage as the base for using additive manufacturing technology in order to deliver the final prototype of the jewelry.

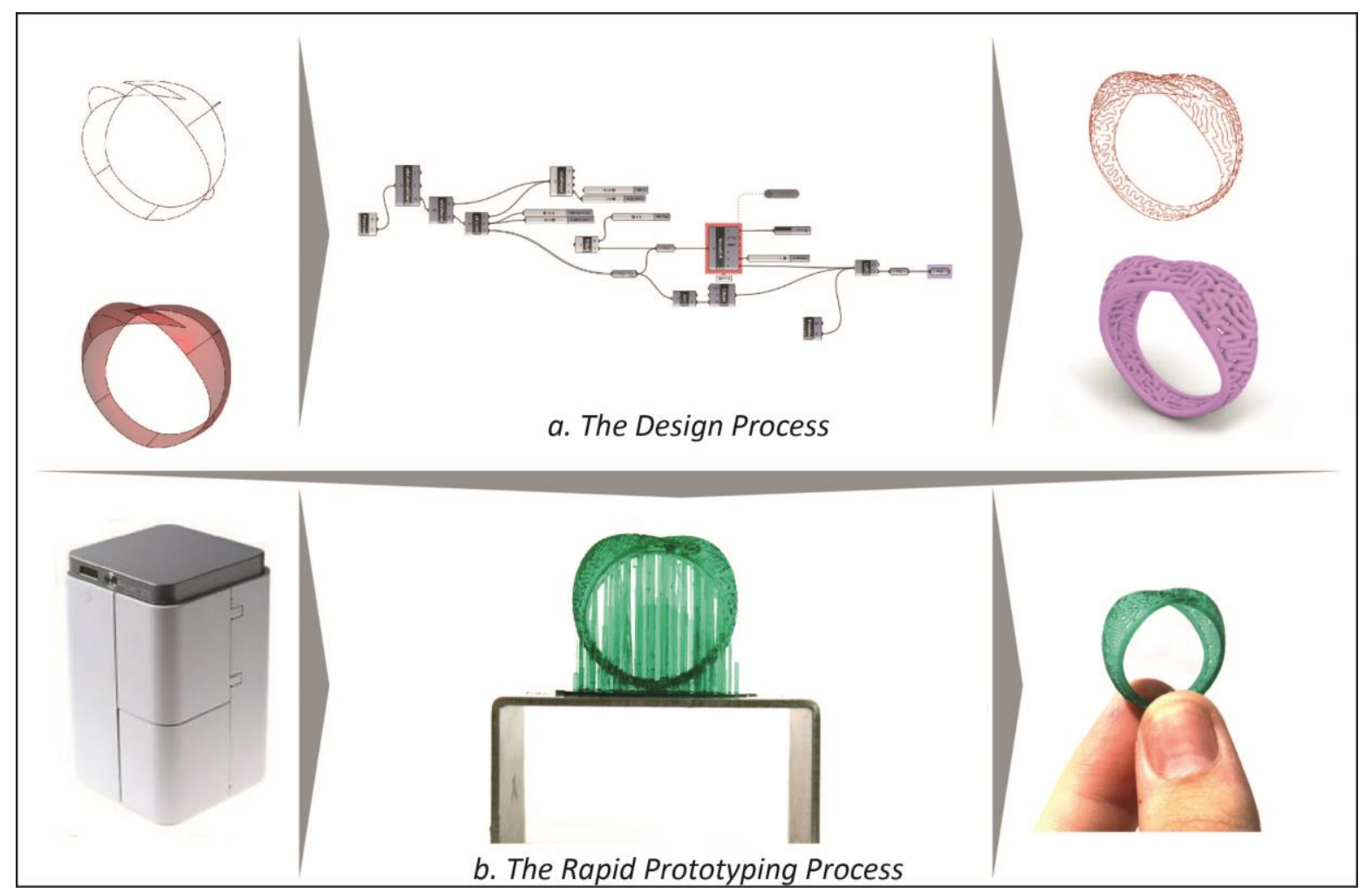

Figure 1: The research workflow applied

\section{METHODS}

Computational design is a modern methodology of using textual or visual programming interface to create and modify forms, geometries even decorations (Kyratsis, 2020; Manavis et al, 2020b). This type of design methodology permits the automation of the design procedures and extends the standard features of CAD applications going beyond their limitations. Computational design is offering several opportunities for novelty and superior solutions as allows to applications to generate structures and deals with digital fabrication tools.

In this research, we used the visual programming of Rhino ${ }^{\mathrm{TM}}$ with a graphical algorithm editor named Grasshopper $^{\mathrm{TM}}$ as the main tool. Grasshopper ${ }^{\mathrm{TM}}$ does not require programming or scripting knowledge, nevertheless, permits designers a high degree of flexibility in creating both simple and complex forms. It is a new way to expand and control the 3D design and modeling processes, involving computerizing repetitive processes, developing forms through mathematical functions, and creating complex forms through repetitions of simple geometry.

In our case, we used the Mobius strip modelling (Figure 2) to design the ring. Grasshopper ${ }^{\mathrm{TM}}$ has extended the mathematical definition of Mobius strip concept to any base geometry that flows along a circle to meet its own end. As we created a new logic structure, we followed three basic steps. The first one was to create a base geometry and a trap to flow along the track. The second step was of course the flow of the trap onto the track. And the third step was the rotation. In our case this is a ring, so we went 
further from the rotation and changed the thickness of the geometry, having different forms of our jewelry. Grasshopper ${ }^{\mathrm{TM}}$ allows us to have many iterations of the product with different geometries easily and fast. These iterations are part of the family of alternative designs that can be produced in a manageable way via the algorithm built and the suggested parameters introduced. The final selection is part of the aesthetics that every designer has and can be associated with the customer demands when a specific trend is followed. In this case, the use of computational design is the basis for creating a new trend with unusual geometries, difficult to manufacture forms and the use of advanced additive manufacturing technology with increased dimensional accuracy.

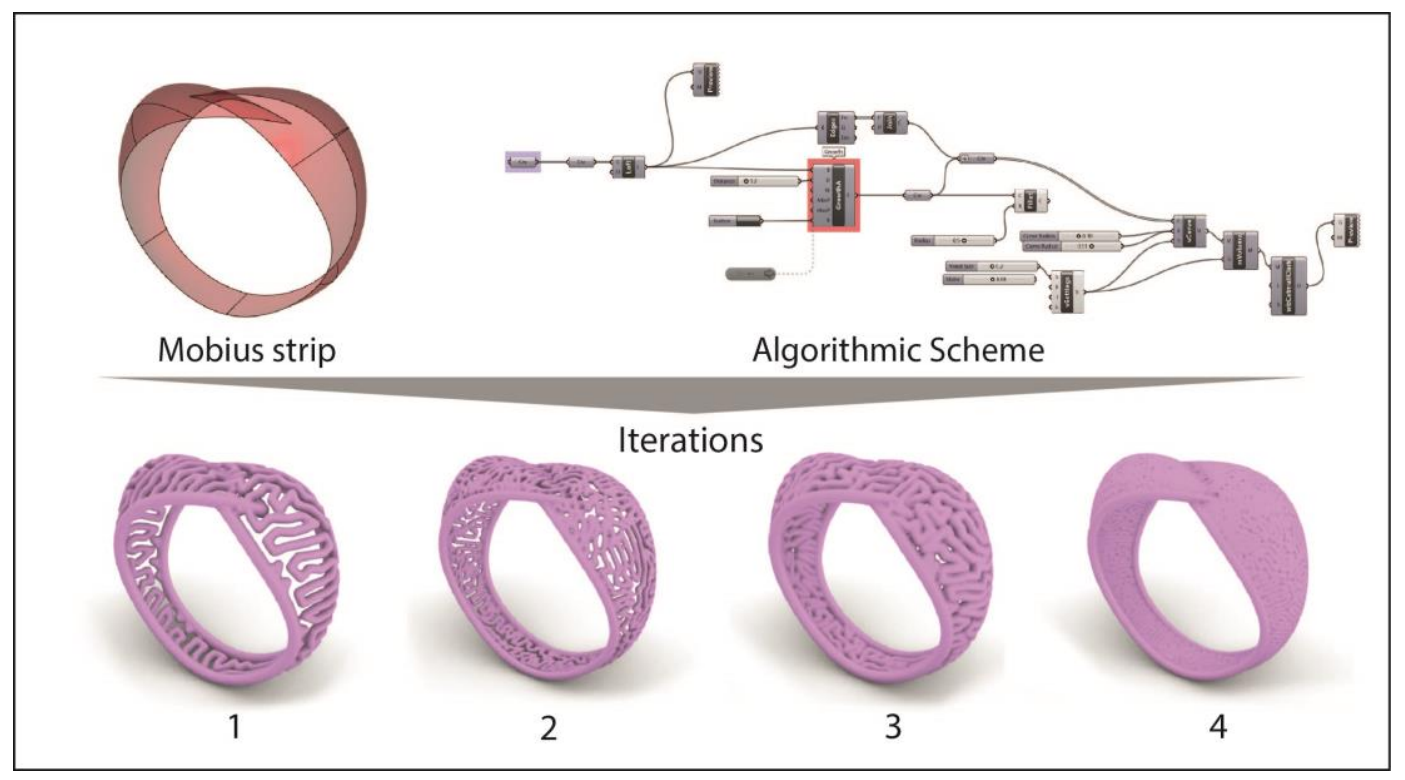

Figure 2: The steps of the design process and iterations

\section{RESULTS}

For the prototype model construction, the ProJet ${ }^{\circledR} 1200$ Micro-SLA, 3D printer from 3D Systems was used. It accepts a variety of file formats (STL, OBJ, PLY, ZPR, ZBD, WRL, 3DS, FBX, IGES, STEP etc), its native resolution is 56 microns and its layer thickness is $0.03 \mathrm{~mm}$. The Micro-SLA technology used is an additive manufacturing process that belongs to the Vat Photopolymerization family and incorporates a number of advantages when the output model has limited dimensions.

The model was created by selectively curing a polymer resin layer-by-layer using an ultraviolet (UV) laser beam. The material used was 3D Systems' VisiJet ${ }^{\circledR}$ FTX Green material. It is a material with high durability and rigidity that is made for plastic models prototyping and small item casting patterns. As a result, it is considered an excellent solution for the jewelry industry. Its tensile strength (ASTM D638) is $30 \mathrm{MPa}$ and its Ash Content is $0.01 \%$. In addition, other industries involved with small sized components can use the same technology.

This printer makes small precise parts with amazing fine features details, even more stunning jewelry casting patterns (Ferreira et al, 2012). The curing area is built into the unit and prints 30-micron layers at 585 dpi resolution with $43 \times 27 \times 180 \mathrm{~mm}^{3}$ print volume area. The smallest area lithography machines developed to produce small but highly detailed objects suitable for a range of applications including casting and jewelry. The use of this specific $3 d$ printer offers us a high precision and exceptionally fine feature detail reflecting real CAD accuracy, it delivers a smooth surface finish, minimizing polishing of precious materials. It is suitable for producing strong, precise micro parts such as figurines scale models of aircraft, fashion accessories etc.

In Figure 3, the rapid prototyping process is illustrated. Once the printing was completed, we removed all the supports from the model. Supports are necessary for successful SLA printing. Removing the marks left by supports becomes an integral step of the finishing process. It can be seen that the model is highly accurate and can be used not only for prototyping purposes but for manufacturing as well. The material used is castable with clean, ash-free burnout and can offer a great deal of accuracy when the final product is built. 


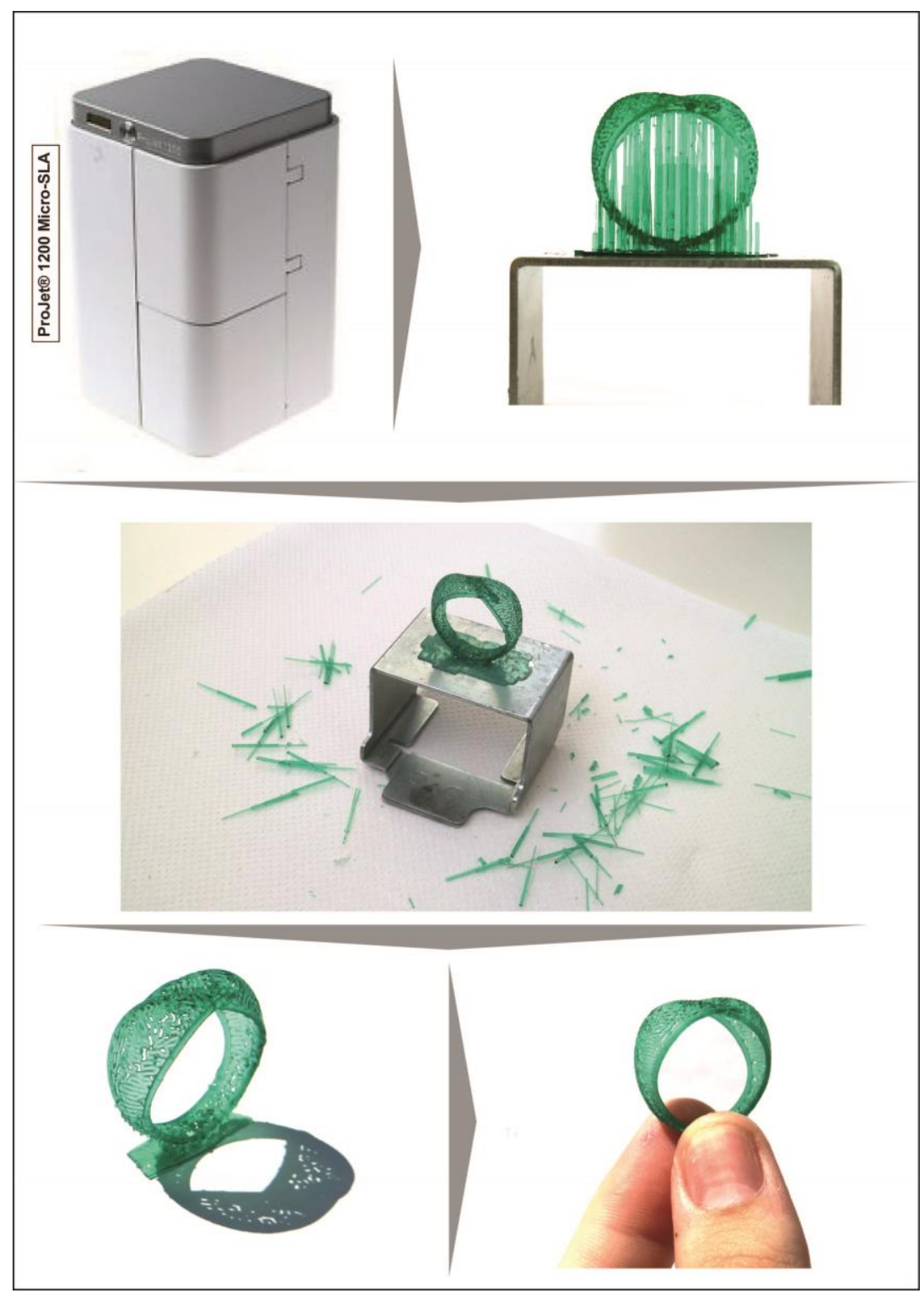

Figure 3: The steps followed during the prototyping process

\section{DISCUSSION}

Computational design allows designers to interact with geometries turned into solid three-dimensional models to develop different forms and versions of the product. The proposed approach can create many patterns on jewelry products, which is difficult and time consuming to be done with the conventional ways. Furthermore, gives the opportunity to present different aesthetic results and even more to improve the fabrication by checking a number of different versions of the product (family of products creation).

Also, there are some additional techniques that can be used in the design process, like the creation of Voronoi diagrams. This kind of techniques gives the designers unlimited geometry variations by offering them the opportunity to play more with shapes and forms. The next step of the research is the development of jewelry based on the traditional styles using Computer Aided Geometric Modeling. The target is the connection of mathematical models and algorithms with traditional patterns in order to design families of jewelry with creative sense, traditional flair and imagination. In such a way, every built algorithm can be used to produce a variety of CAD forms that can lead to alternative proposals of the product designed. These forms are used later on as the base for finalizing the product itself. Even then, more designs can be produced be altering the parameters introduced into the algorithm and thus extra inspiration, design and manufacturing capabilities can be triggered. 


\section{CONCLUSIONS}

In this paper, a computer-aided production approach for jewelry design technique is presented. It is an effort to exploring new approaches of designing and manufacturing ornamental products, using mathematical and modern CAD tools. We highlighted the potential for the design of complex geometric forms and their control during the whole process. Through computational design we generated different forms effectively with a structured manner. The examined automatic digital fabrication doesn't try to cancel the hand manipulation of physical materials, on the contrary it recommends a chance for exploration new craft-making tools and practices from a more modern point of view, showing new opportunities for digital and handmade practices.

The use of Grasshopper ${ }^{\mathrm{TM}}$ makes the digital system more user-friendly and facilitate the manipulation of complex conditions. This CAD tool allows designers to make efficient the iterative design process and enable different products in many geometries, sizes and types, while shortening simultaneously the necessary time for creating models. Finally, the usage of the ProJet ${ }^{\circledR} 1200$, 3D printer makes the final prototype aesthetically perfect as it is suitable for producing strong fashion accessories and jewelry.

\section{ACKNOWLEDGMENTS}

The authors would like to thank the Direction Team of the CODE + Laboratory in the University of Western Macedonia, for its valuable help, making available human and technical resources.

\section{REFERENCES}

[1] Ferreira, T., Almeida, H-A., Bártolo, P-J., Campbell, I.: "Additive Manufacturing in Jewellery Design”, $11^{\text {th }}$ Biennial Conference on Engineering Systems Design and Analysis 2012, (ASME 12, Nantes, France, 2012).

[2] Goel, V., Khanduja, D., Garg, T.K., Tandon, P.: "Computational Support to Design and Fabrication of Traditional Indian Jewelry", Computer-Aided Design and Applications 12(4), 457-464, 2015. doi: 10.1080/16864360.2014.997642.

[3] Iarussi, E., Li, W., Bousseau, A.: "Wraplt: computer-assisted crafting of wire wrapped jewelry", Journal ACM Transactions on Graphics 34(6), 2015. doi: 10.1145/2816795.2818118.

[4] Kyratsis, P.: "Computational design and digital manufacturing applications", International Journal of Modern Manufacturing Technologies 12(1), 82-91, 2020.

[5] Manavis, A., Minaoglou, P., Aidinli, K., Efkolidis, N., Kyratsis, P.: "CAD based design for the jewellery industry: A case study", Proceedings of Computing and Solutions in Manufacturing Engineering 2020a, (COSME, Brasov, Romania, 2020).

[6] Manavis, A., Minaoglou, P., Tzetzis, D., Efkolidis, N., Kyratsis, P.: “Computational design technologies for interior designers: a case study", Proceedings of Computing and Solutions in Manufacturing Engineering 2020b, (COSME, Brasov, Romania, 2020).

[7] Miguel, E., Lepoutre, M., Bickel, B.: "Computational Design of Stable Planar-Rod Structures", Journal ACM Transactions on Graphics 35(4), 2016. doi: 10.1145/2897824.2925978.

[8] Shen, Y., Zhang, T., Ke, Y., Wang, H.: "Design and application of three-dimensional parametric technology in construction of new forms of modern clothing", Journal of Textile Research 39(12), 118-123, 2018.

[9] Silina, Y., Haddadi, H.: "New directions in jewelry: a close look at emerging trends \& developments in jewelry-like wearable devices", Proceedings of the ACM International Symposium on Wearable Computers 2015, (ISWC, New York, USA, 2015).

[10] Somlak, S.: "Reviews of Computer-Aided Technologies for Jewelry Design and Casting", Naresuan University Engineering Journal 1(6), 41-56, 2011. doi: 10.14456/nuej.2011.8.

[11] Tian, Y., Miao, Y., Yu, Y., Zhang, Z.: "Parametric Design Method Based on Grasshopper and Shoe Last Bottom Pattern Moulding Characteristics", IOP Conference Series: Materials Science and Engineering, 520(1): 012017, 2019. doi: 10.1088/1757-899X/520/1/012017.

[12] Wang, Y., Yang, X., Fukusato, T., Igarashi, T.: "Computational Design and Fabrication of 3D Wire Bending", Proceedings of the SIGGRAPH 2019, (Asia SA, 2019), doi: 10.1145/3355056.3364575. 


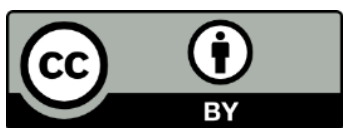

(C) 2020 Authors. Published by the University of Novi Sad, Faculty of Technical Sciences, Department of Graphic Engineering and Design. This article is an open access article distributed under the terms and conditions of the Creative Commons Attribution license 3.0 Serbia

(http://creativecommons.org/licenses/by/3.0/rs/). 